\title{
Revitalisation urbaine et gentrification positive : le cas du Cinéma Beaubien à Rosemont ${ }^{1}$
}

\author{
Wilfredo Angulo ${ }^{a}$, Juan-Luis Klein ${ }^{b}$, Diane-Gabrielle Tremblay
}

RÉSUMÉ. Ce texte analyse le cas du Cinéma Beaubien à Rosemont, un projet culturel soutenu par une coalition d'organisations de la société civile. Grâce à l'élaboration de stratégies inclusives favorisant la cohésion sociale et la vitalité locale, ce projet a dynamisé une artère commerciale en friche et a été central dans la revitalisation récente du quartier. Ce cas nous permet de mieux saisir l'effet des projets culturels portés par des organismes communautaires, ancrés localement et encastrés dans un modèle économique où se conjuguent les échelles et les acteurs. L'ancrage local facilite l'émergence de nouvelles aspirations mobilisant les citoyens et développe le sentiment d'appartenance au territoire. Cette recherche ouvre la voie à de nouvelles interprétations postulant que, sous certaines conditions, les projets culturels peuvent éviter les effets négatifs associés à la gentrification, souvent concomitants à la revitalisation d'un milieu, lorsqu'ils sont amorcés par l'action collective et soutenus par du leadership partagé.

\begin{abstract}
This text analyses the case of Cinema Beaubien in Rosemont, a cultural project supported by a coalition of civil society oryanizations. Through the development of inclusive strategies for social cohesion and local vitality, this project has energized a devitalized commercial area and is contributing to the recent revitalization of the neighbourbood. This case allows us to better understand the effect of cultural projects carried out by community organizations, rooted locally and embedded in an economic model where scales and actors combine. Local anchoring facilitates the emergence of new aspirations that mobilize citizens and develops a sense of belonging to the territory. This research opens the door to new interpretations postulating that, under certain conditions, cultural projects can avoid the negative effects associated with gentrification, often associated with the revitalization of an environment, when they are initiated by collective action and supported by shared leadership.
\end{abstract}

\section{Introduction}

Cet article porte sur le rôle des initiatives culturelles dans les démarches de revitalisation urbaine portées par des organismes communautaires. Nous postulons que, sous certaines conditions, le choix de la culture peut agir comme catalyseur favorisant l'élaboration de stratégies inclusives qui ont un effet positif sur le territoire en matière d'activité économique et de milieu de vie, et qui renforcent la participation citoyenne, la cohésion sociale et la vitalité locale. Plus concrètement, nous analysons le cas du Cinéma Beaubien, que nous plaçons dans le contexte de la revitalisation du quartier Rosemont.
La relance de ce cinéma de quartier a été menée par une coalition d'acteurs dans laquelle la Corporation de développement économique communautaire de Rosemont-Petite-Patrie (CDEC-RPP) a assuré un très fort leadership. Notre hypothèse repose justement sur le fait que ce processus a été amorcé par l'action collective citoyenne, ce qui expliquerait pourquoi nous ne voyons pas dans ce cas les effets négatifs associés habituellement aux expériences de revitalisation impulsées par des initiatives culturelles, lesquelles conduisent souvent au remplacement des populations résidentes par des populations économiquement plus aisées. Nous montrerons que, au contraire, le Cinéma Beaubien a été un facteur

\footnotetext{
a Post-doctorant, Université du Québec à Montréal

b Professeur titulaire, département de géographie, Université du Québec à Montréal

c Professeure titulaire, Université Téluq
} 
important de revitalisation et de revalorisation locale, et que la majorité des citoyens se voient comme une partie prenante de l'action collective liée à cette entreprise dont ils se sentent fiers puisque des actions collectives les ont associés à cette revitalisation.

L'étude de cas est présentée en six étapes. En premier lieu, nous présentons brièvement la problématique du développement local par la culture. En deuxième lieu, nous présentons la méthodologie de la recherche sur la relance du Cinéma Beaubien. En troisième lieu, nous situons cette initiative dans le cadre du développement communautaire à Rosemont, notamment en lien avec la CDEC de Rosemont-Petite-Patrie. En quatrième lieu, nous présentons la perception que les acteurs locaux et les citoyens ont de la contribution du cinéma au développement de leur quartier. En cinquième lieu, nous abordons le dynamisme insufflé dans le quartier grâce à la relance du cinéma. Enfin, en sixième lieu, nous interrogeons l'expérience de revitalisation en regard des dangers de gentrification.

\section{L'option de la culture dans la revitalisation urbaine}

Pour bien situer le contexte historique de la relance du Cinéma Beaubien, il faut rappeler que les anciens quartiers industriels de Montréal, dont Rosemont, ont traversé, à partir des années 1980, une crise économique sévère qui a provoqué leur dévitalisation sur les plans productifs, commerciaux et démographiques (Klein et Shearmur, 2017; Tremblay, Klein et Fontan, 2016). Le phénomène de la mondialisation de l'économie a affecté sévèrement ce type de quartier dans l'ensemble des pays dits industrialisés (Fontan, Klein et Lévesque, 2003). L'effondrement de la base économique de ces quartiers a souvent été brutal, mais, en même temps, dans certains cas, les acteurs socioéconomiques de ces quartiers ont réussi à mettre en œuvre des projets alternatifs portés par la société civile et par des organismes associés à l'économie sociale et solidaire. Cela a toutefois obligé ces acteurs à innover en ce qui concerne autant les modalités d'action que la gouvernance des initiatives mises en œuvre.

Plusieurs travaux soutiennent que, dans la société qui émerge de la crise de la société industrielle, le territoire et ses ressources culturelles peuvent prendre une place importante dans les stratégies de développement, stratégies qui visent la différenciation des territoires afin d'accroitre leur compétitivité dans l'espace mondialisé (Scott et Leriche, 2005; Ambrosino et Andres, 2008; Andres, 2010; Andres et Grésillon, 2011). La concurrence pour attirer des investissements et des populations susceptibles de favoriser la reprise économique a mené plusieurs villes à mettre de l'avant des stratégies culturelles (Darchen et Tremblay, 2013; Scott, 2014). Des instances gouvernementales ainsi que des institutions privées et publiques se sont engagées dans la création de conditions propices pour rendre plus attractives leur ville et leur région en faisant de grands investissements afin de mieux les équiper pour faire face à la concurrence mondiale des territoires, à travers une stratégie de marketing territorial axée sur les actifs culturels.

Dans ce contexte, les politiques culturelles sont conçues comme des outils de restructuration urbaine et de développement économique. Les différentes stratégies déployées (district culturel, tourisme, événements culturels médiatisés, équipements et infrastructures), que ce soit en matière d'aménagement du territoire ou de développement touristique, sont mobilisées pour la promotion des villes (Hall, 2000; Scott, 2000; Hutton, 2008). Cependant, en général, ces stratégies s'inscrivent dans une approche marchande et parfois élitiste (grandes institutions musicales, musées, etc.) de la culture, qui évacue les questions d'ordre social et d'accès à la culture. Or, la prise de conscience des enjeux culturels par les acteurs locaux, notamment les acteurs communautaires, accentue et renforce l'attention accordée au milieu local et, de ce fait, au territoire, puis ramène la place et le rôle des résidents au centre des projets (Klein, Tremblay, Sauvage, Ghaffari et Angulo, 2019).

Des effets de gentrification peuvent avoir lieu dans la foulée des interventions culturelles dans les quartiers en raison de l'incapacité des résidents plus démunis d'assumer les conséquences de la hausse des prix fonciers et des changements de services offerts par la structure commerciale ou par les organismes présents. Dans ces cas, les résidents de longue date sont progressivement évincés du quartier par l'arrivée de résidents plus riches, «les gentrificateurs »(Glass, 1963; Ley, 1994; Smith, 1996). Par contre, des travaux ont montré que des 
modalités de gentrification socialement acceptables sont possibles (Ghaffari, Klein et Angulo Baudin, 2018). C'est ainsi que plusieurs auteurs (RiusUlldemolins et Posso-Jiménez, 2016; Janoschka et Sequera, 2014; Díaz-Parra et Rabasco Pozuelo, 2013; Boldrini et Malizia, 2014; Sabatini, DarellaRoble et Vásquez, 2009) donnent à voir des processus par lesquels les initiatives culturelles non seulement ne provoquent pas l'éviction des résidents, mais valorisent des quartiers marginalisés et stigmatisés, et peuvent avoir un effet positif, y compris sur les populations moins bien nanties (Ghaffari, 2020).

\section{Méthodologie}

Notre recherche est centrée sur l'étude de la relance du Cinéma Beaubien et sur les effets de cette initiative sur son territoire. Ce territoire se situe dans l'arrondissement Rosemont-La Petite-Patrie, qui compte 134000 habitants, ce qui correspond à 8,1\% de la population totale de la ville de Montréal, et s'étend sur un territoire de 15,9 $\mathrm{km}^{2}$. De façon plus précise, nous avons établi un périmètre englobant une zone influencée de façon plus directe par le cinéma, qui est délimitée par la 3e Avenue à l'est, par la rue de Bordeaux à l'ouest, par la rue Saint-Zotique au nord et par la rue de Bellechasse au sud (voir figure 1). La zone mesure $561330 \mathrm{~m}^{2}$, comprend 2095 résidences et compte 3770 résidents, parmi lesquels $37 \%$ sont propriétaires et $63 \%$ sont locataires.

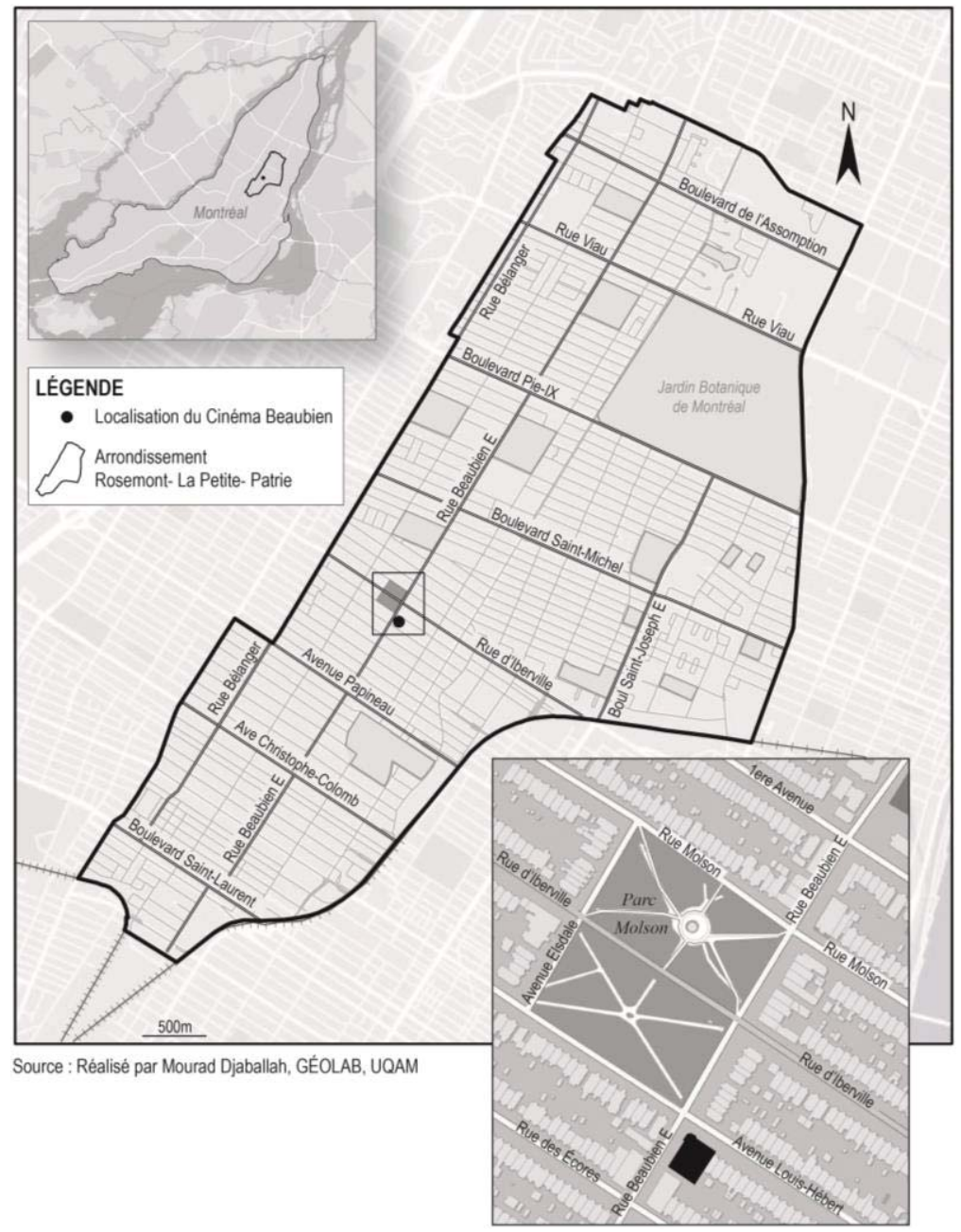

Figure 1 - Le Cinéma Beaubien à Rosemont Source : Ville de Montréal, modifiée par les auteurs 
La recherche a été menée par une consultation de documents et, principalement, par la réalisation d'entrevues semi-directives. Nous avons interviewé des répondants provenant des organismes concernés par notre cas, soit des représentants de la Corporation du Cinéma Beaubien, des tables de quartier sur la culture, des groupes culturels, des associations communautaires, des organismes de développement économique et des organismes de l'économie sociale du quartier. Nous avons interviewé aussi des représentants des instances politiques (élus et fonctionnaires), des directeurs d'entreprise et des citoyens. Dans l'ensemble, nous avons interviewé 26 personnes, dont les trois quarts, en plus de représenter leur organisme, résidaient ou avaient résidé jusqu'à récemment dans la zone étudiée. Les répondants ont été choisis en fonction de leur expertise et expérience ainsi que de la pertinence de leur organisme par rapport à l'objet d'étude. Les entrevues ont été réalisées entre septembre 2016 et juillet 2017. Elles ont été enregistrées et leur contenu a été analysé à l'aide d'une grille construite à partir de notre guide d'entretien.

\section{Le développement communautaire : base organisationnelle de la relance du Cinéma Beaubien}

Il faut préciser dès le départ que la Corporation de développement communautaire de RosemontPetite-Patrie (CDEC-RPP) a été l'organisme leader du processus de relance du Cinéma Beaubien ${ }^{2}$. Cet organisme avait une longue expérience en mobilisation pour la relance économique des quartiers, expérience qui remontait à son intervention dans la reconversion du terrain laissé en friche par la fermeture des Ateliers Angus de la compagnie Canadian Pacific Rail en 1992 (Fontan, Klein et Tremblay, 2005). Depuis la reconversion des terrains des Ateliers Angus, plusieurs projets ont mobilisé la CDEC-RPP, favorisant ainsi le sentiment d'appartenance des acteurs sociaux locaux ainsi qu'une fierté collective (Klein et Morrissette, 2014). Le capital social des acteurs du territoire s'est vu renforcé, ce qui a augmenté leur capacité de mettre en œuvre de nouveaux projets de revitalisation dans le territoire. La CDEC y a été pour beaucoup dans cette valorisation de la capacité collective de la population locale.
La relance du Cinéma Beaubien résulte d'une réaction à la fermeture du Cinéma Le Dauphin en 2000 par la chaine Loews Cineplex Odeon, qui en était propriétaire. Cette fermeture venait ajouter un maillon de plus à l'ensemble des éléments qui ont provoqué la dévitalisation du quartier à partir des décennies 1980 et 1990. Étant donné que ce cinéma constituait un lieu culturel significatif pour la population de Rosemont et un élément important pour la vitalité des commerces de la rue Beaubien, les résidents et les commerçants du quartier se sont mobilisés pour empêcher sa fermeture. Le projet a reçu le soutien du public, du milieu communautaire et du milieu de la diffusion cinématographique. Il a été soutenu par la CDEC-RPP, qui a entrepris des démarches, dès 2001, afin d'acheter et de sauvegarder le cinéma (Tremblay, Klein et Rochman, 2014).

La mobilisation par la CDEC des réseaux de financement de l'économie sociale ainsi que son capital de reconnaissance obtenu grâce à ses interventions pour favoriser le développement local depuis sa création en 1990 ont donné une grande force à son appui au projet, ce qui a permis de recueillir les fonds et les ressources humaines nécessaires pour la reprise du cinéma. La négociation directe avec les acteurs politiques a permis la mise en œuvre d'un programme conçu spécifiquement pour le projet. Le financement total amassé a été de 1,4 million de dollars. Le nouveau Cinéma Beaubien est devenu une entreprise d'économie sociale très ancrée dans la communauté locale. Son conseil d'administration est formé de sept personnes, dont trois représentent différents secteurs socioéconomiques du quartier. La CDEC y était représentée par deux membres, jusqu'à son abolition en 2015.

Le Cinéma Beaubien a tissé de nombreux liens avec la communauté d'acteurs qui interviennent dans le territoire dans diverses facettes du développement local. Le directeur du cinéma est présent dans différentes instances locales. Il participe aux tables vouées au développement culturel du quartier, mais aussi aux tables et aux regroupements d'acteurs du développement économique et social en général. Il est présent aussi dans les différents forums qui se tiennent dans les milieux d'intervention sur la culture et le développement local.

Le cinéma compte sur des ressources humaines et organisationnelles diversifiées provenant aussi bien du milieu local que de l'ensemble de la Ville de 
Montréal. En ce qui concerne les ressources financières, il consolide de plus en plus son autonomie. Sa programmation, offerte dans cinq salles, est considérée comme culturellement enrichissante pour la communauté locale. De plus, la direction de l'entreprise organise des activités culturelles en partenariat avec des institutions d'enseignement, des centres de la petite enfance, des salles de spectacles et des festivals. Le cinéma est ainsi à la fois un lieu de diffusion et d'animation culturelle important et reconnu par la collectivité :

Le Cinéma Beaubien, c'est comme une institution, c'est comme une histoire. Les gens l'aiment cette histoire-là. C'est une belle réussite, ça été un bon travail. Des fois, ce n'est pas clair, les gens se demandent : à qui appartient le cinéma? Est-ce que c'est un OBNL? Mais les gens savent que ce cinéma-là a été récupéré avec la force des citoyens, il y a eu des pétitions. C'est une histoire de quartier. Moi, je dis souvent à Mario [Mario Fortin, le directeur général du cinéma] : «Quand le cinéma tousse, tout le monde s'enrhume. » Ça fait vraiment le poumon du quartier. (Entrevue no 19 , résident, 2017)

Ainsi, la CDEC et la mobilisation communautaire ont transformé une entreprise en faillite en une structure rentable et profitable, tant sur le plan du maintien et de la création d'emplois que, comme nous tenterons de le montrer, sur celui des bénéfices pour l'économie du quartier.

\section{Le Cinéma Beaubien, principal repère identitaire des résidents et des acteurs locaux}

Un des objectifs de notre recherche a été de connaitre l'intensité de l'ancrage que le Cinéma Beaubien a pu développer dans le territoire local, et de vérifier à quel point les résidents et l'ensemble des acteurs du quartier s'identifient au cinéma en tant que jalon du développement de la collectivité. D'abord, il faut dire que, lorsque nous avons demandé aux résidents quels étaient les acteurs majeurs du quartier, le Cinéma Beaubien est ressorti comme l'un des plus importants, voire comme le principal repère identitaire des résidents, notamment à cause de l'image positive du quartier que le cinéma véhicule. Les entrevues réalisées nous indiquent que la présence du cinéma a eu un effet de renforcement sur le sentiment d'identité et d'appartenance au quartier, lequel était déjà très fort.

\section{L'effet de levier culturel du Cinéma Beaubien}

Le Cinéma Beaubien a inspiré de nouveaux projets dans le quartier qui se sont traduits par l'intensification de l'activité culturelle et socioéconomique. Cet effet s'est manifesté à travers les capacités que le projet a insufflées aux différents acteurs locaux concernant le développement culturel et l'amélioration des conditions de vie des résidents. En effet, un des répondants rapporte :

Le Cinéma Beaubien est un de nos lieux culturels forts dans notre territoire, mais ce n'est pas juste parce que c'est un lieu culturel. Le Cinéma Beaubien redimensionne tout le quartier. J'habite le quartier depuis 2001 et j'ai vu le quartier se transformer. [...] Moi, je le vois, la vie de quartier, les commerces, tout ce qui se développe autour. Il y a eu une transformation vraiment importante dans le quartier. (Entrevue $\mathrm{n}^{\circ} 2$, acteur culturel, 2017)

Les répondants soulignent la présence du Cinéma Beaubien comme un facteur de vitalité culturelle de proximité. Récemment, le cinéma s'est doté d'équipements numériques, ce qui lui permet d'être compétitif dans un marché très concurrentiel. Il vient d'investir plusieurs milliers de dollars pour acquérir une technologie permettant aux personnes sourdes et malentendantes d'avoir accès au cinéma en salle et de suivre les sous-titres d'un film sur un capteur individuel. Il s'agit d'un premier pas pour accroitre encore plus l'accessibilité à la culture. De plus, le cinéma sert de salle d'exposition pour des œuvres d'art. Il développe aussi des activités de médiation culturelle, en partenariat avec l'arrondissement et la Maison de la culture. Le cinéma participe également à divers festivals, dont le Festival international du film pour enfants de Montréal (FIFEM) et le Festival international Vues d'Afrique. Par ailleurs, le cinéma est gestionnaire du piano public installé dans le parc Molson, activité soutenue par l'association des commerçants et par la mairie d'arrondissement.

L'effet du cinéma déborde largement le champ de la culture. En tant qu'initiative de développement local, le cinéma a évolué de concert avec le quartier. Il assure un leadership qui s'étend à divers secteurs et organismes, à travers différentes collaborations et alliances. Il est au centre d'un vaste réseautage organisationnel facilité par la participation de ses 
responsables dans des tables de concertation et dans les conseils d'administration de divers organismes locaux. Le fort engagement du Cinéma Beaubien dans le quartier donne à voir un ancrage territorial important. Il a approfondi et densifié ses actions dans le territoire à travers de nouveaux projets innovateurs, et bénéficie d'une forte reconnaissance auprès des acteurs et des résidents. Ceux-ci sont fiers de la présence du cinéma et valorisent le fait d'avoir réussi la sauvegarde d'un élément du patrimoine culturel et, ainsi, d'avoir participé à l'empowerment collectif, c'est-à-dire à la capacité de coordonner des actions et d'agir collectivement.

Pour synthétiser la perception des acteurs au sujet de la présence du Cinéma Beaubien dans le quartier, nous proposons un tableau indiquant les réponses obtenues des intervenants, des commerçants et des résidents concernant le rôle du cinéma (voir tableau 1).

\begin{tabular}{|l|l|}
\hline Acteurs & \multicolumn{1}{c|}{ Perceptions } \\
\hline $\begin{array}{l}\text { Intervenants com- } \\
\text { munautaires et insti- } \\
\text { tutionnels }\end{array}$ & $\begin{array}{l}\text { - C'est une icône de la mobilisation citoyenne. } \\
\text { - Produit partie du quartier. }\end{array}$ \\
& $\begin{array}{l}\text { - Le cinéma est positif pour le commerce. } \\
\text { - Les restaurants vendent plus grâce au cinéma. } \\
\text { - Représente la lutte citoyenne et l'attachement au quartier. }\end{array}$ \\
\hline Commerçants & $\begin{array}{l}\text { - Présente des films de qualité. } \\
\text { - Contribue à l'image positive du quartier. }\end{array}$ \\
& $\begin{array}{l}\text { - Représente la lutte citoyenne et l'attachement au quartier. } \\
\text { - Il y a une belle histoire derrière le cinéma. }\end{array}$ \\
& $\begin{array}{l}\text { - C'est un moteur de la culture. } \\
\text { - C'est une belle réussite. } \\
\text { - C'est le poumon du quartier. }\end{array}$ \\
& $\begin{array}{l}\text { - C'est 100 \% nécessaire. } \\
\text { - C'est le cœur du quartier. }\end{array}$ \\
\hline Résidents & $\begin{array}{l}\text { - C'est une icône du lieu. } \\
\text { - C'est un repère identitaire. } \\
\text { - C'est une référence culturelle. }\end{array}$ \\
\hline
\end{tabular}

Tableau 1 - Perception de la présence du Cinéma Beaubien dans Rosemont Source : Angulo, 2019, p. 101-102

\section{Un risque de gentrification?}

Dans cette section, nous nous interrogeons sur la perception des acteurs concernant l'éventuel effet gentrificateur du Cinéma Beaubien sur le territoire de Rosemont, en particulier sur sa zone d'influence plus directe. Il ressort de l'analyse des propos des répondants sur les changements dans le quartier qu'ils n'ont pas de craintes concernant d'éventuels effets négatifs que le cinéma pourrait entrainer. Les résidents sont heureux dans le quartier, ils aiment leur quartier et ils soulignent qu'il n'y a aucune action contre le développement d'initiatives culturelles. Ils nous assurent qu'il n'y a pas d'actes de vandalisme sur les bâtiments ni sur les commerces, comme cela s'observe dans d'autres quartiers où les initiatives culturelles sont contestées par les citoyens (Ghaffari, 2020). Au contraire, les résidents s'engagent dans les améliorations du quartier. Parmi les commerçants interviewés, certains ont fait des rénovations, d'autres ont agrandi et diversifié leur commerce, mais aucun n'a changé de secteur d'activité.

\subsection{Les nouveaux résidents : des gentrificateurs potentiels?}

Selon nos répondants, les résidents qui arrivent dans le quartier sont des gens qui adhèrent à l'identité de celui-ci, qui connaissent son histoire et qui sont intéressés par elle. On ne constate donc pas le cas classique de remplacement des couches populaires par des couches sociales plus aisées, qui amènent avec elles de nouveaux modes de vie et des valeurs différentes. D'ailleurs, les nouveaux résidents interviewés affirment que leur changement résidentiel était motivé par la recherche de conditions pour la vie familiale et par les caractéristiques du quartier tel qu'il est. Quant aux résidents de longue date participant à nos entrevues, ils n'ont 
pas fait référence à des attitudes de rejet à l'endroit des nouveaux résidents. Au contraire, ceux-ci sont très appréciés pour ce qu'ils apportent en matière de ressources économiques et professionnelles. On pourrait considérer qu'ils renforcent le capital socioterritorial du quartier.

Néanmoins, il y a eu des augmentations du prix des propriétés et du mètre carré du terrain dans le quartier. Bien sûr, certaines de ces augmentations peuvent être attribuables à la présence du cinéma. Toutefois, ces augmentations ne s'écartent pas de la tendance générale qu'affiche la métropole. Par ailleurs, d'après les entrevues, les résidents ne semblent pas affectés par ces augmentations, et ce, à cause de la part importante des résidents propriétaires. Ceux-ci rapportent que les augmentations des évaluations foncières représentent une reprise de la valeur de leur patrimoine immobilier par rapport à la dévalorisation provoquée par la crise des années 1980-1990. De plus, cela leur assure des revenus pour l'avenir, éventuellement pour la retraite, une plus-value qu'ils ne sont pas prêts à encaisser pour le moment, car ils se sentent très bien dans le quartier qu'ils habitent.

Les répondants nous ont parlé d'un rajeunissement de la population du quartier grâce à l'arrivée de jeunes familles, sans pour autant que cela ne déplace la population des aînés. Les répondants indiquent aussi que cela se fait graduellement, sans chasser personne, les aînés continuant ainsi à vivre une vie de quartier qui leur convient très bien. Ce caractère graduel du changement générationnel est confirmé par les données statistiques sur la mobilité résidentielle : les données analysées (Angulo, 2019, p. 120-121) révèlent une très grande stabilité résidentielle de la population dans le quartier. Cette constatation est validée par la stabilité de la moyenne d'âge du quartier, qui se maintient depuis 15 ans autour de 39 ans. Par contre, les résidents ont des craintes quant à la difficulté pour leurs enfants d'acheter une propriété et de rester dans le quartier.

Les répondants mentionnent aussi que, même si les résidents (y compris les locataires) ne partent pas, l'augmentation du prix des logements peut avoir des conséquences. Les locataires sont prêts à assumer un certain niveau d'augmentation, mais cela se ressent sur leur revenu disponible :
Les gens font un calcul : oui, ça va me coûter plus cher de loyer, mais j'aime mon quartier. Tout est proche et j'ai mes habitudes. Si je déménage, est-ce que je vais trouver quelque chose de moins cher et à peu près pareil? (Entrevue no 3, acteur communautaire, 2017)

Un autre aspect soulevé par ce répondant, ce sont les changements sur le plan des commerces. Il indique que la présence de quelques nouveaux commerces n'a pas d'impact significatif sur la gentrification à Rosemont. Néanmoins, il affirme que le remplacement des petits commerces par des magasins avec des nouveaux produits plus chers pourrait avoir une influence sur la gentrification (Entrevue $n^{\circ} 3$, acteur communautaire, 2017). Le tableau 2 résume les perceptions des acteurs locaux toutes catégories confondues sur la menace de gentrification dans la zone d'étude.

Nos analyses révèlent que le nombre de diplômés ayant un niveau de scolarité postsecondaire dans la zone d'influence du cinéma a augmenté. Le domaine d'activité des résidents est en voie de tertiairisation, et la part de l'emploi dans les secteurs de la culture et de l'enseignement augmente. Cependant, nous constatons aussi que l'évolution des professions des résidents de Rosemont est similaire à celle de l'ensemble de la ville. Comme dans d'autres quartiers, notamment dans ces territoires qui ont eu un passé ouvrier, l'emploi a évolué vers des secteurs de services plus rémunérateurs (Klein et Shearmur, 2017). Nous assistons donc à un processus de « reconversion » (Fontan, Klein et Tremblay, 2005), voire de revitalisation d'un quartier qui avait connu un fort déclin lors de la crise des années 1980, plutôt qu'à la formation d'une enclave de nouveaux résidents susceptibles de construire une nouvelle «identité sociale » (Collet, 2008). Il faut rappeler que si Rosemont comptait une part importante de population ouvrière, ce quartier était déjà aussi composé d'une «classe moyenne » et c'est en quelque sorte un retour à la santé économique qu'il avait connue avant la crise des années 1980, ce qui peut expliquer l'absence de contestation de la revitalisation, plutôt perçue comme un retour au niveau social d'avant la crise. 


\begin{tabular}{|c|c|c|c|c|c|}
\hline Acteurs & $\begin{array}{c}\text { Changements } \\
\text { démographiques }\end{array}$ & $\begin{array}{c}\text { Augmentation du } \\
\text { prix des } \\
\text { propriétés }\end{array}$ & $\begin{array}{c}\text { Changements } \\
\text { dans les } \\
\text { commerces }\end{array}$ & Effets positifs & Menaces \\
\hline $\begin{array}{l}\text { Intervenants } \\
\text { communautaires } \\
\text { et institutionnels }\end{array}$ & $\begin{array}{l}\text { - Les aînés restent } \\
\text { dans le quartier } \\
\text { - Le rajeunissement } \\
\text { du quartier }\end{array}$ & $\begin{array}{l}\text { - Il y a plus de } \\
\text { propriétaires } \\
\text { Transformation } \\
\text { des duplex et tri- } \\
\text { plex en maisons } \\
\text { unifamiliales } \\
\text { Augmentation de } \\
\text { la valeur poten- } \\
\text { tielle des locaux }\end{array}$ & $\begin{array}{l}\text { - Les commerces } \\
\text { s'adaptent à une } \\
\text { nouvelle clientèle } \\
\text { - Les locaux sont } \\
\text { très concurrencés } \\
\text { - Des appartements } \\
\text { ont été convertis } \\
\text { en commerces } \\
\text { - Les commerces } \\
\text { deviennent plus } \\
\text { verts } \\
\text { - Augmentation } \\
\text { des chiffres } \\
\text { d'affaires }\end{array}$ & $\begin{array}{l}\text { Il n'y a pas de } \\
\text { remplacement des couches } \\
\text { sociales par d'autres } \\
\text { Les nouveaux arrivants } \\
\text { apportent de la valeur au } \\
\text { quartier } \\
\text { Intégration sociale }\end{array}$ & $\begin{array}{l}\text { Le quartier } \\
\text { peut devenir } \\
\text { trop bourgeois, } \\
\text { plus } \\
\text { individualiste } \\
\text { Il peut y avoir } \\
\text { un basculement } \\
\text { vers le négatif }\end{array}$ \\
\hline Commerçants & $\begin{array}{l}\text { - Clientèle variée } \\
\text { - Rajeunissement } \\
\text { de la clientèle }\end{array}$ & $\begin{array}{l}\text { - Augmentation de } \\
\text { la valeur } \\
\text { patrimoniale } \\
\text { - Ce sont les prix } \\
\text { normaux des } \\
\text { propriétés à } \\
\text { Montréal }\end{array}$ & $\begin{array}{l}\text { - Augmentation du } \\
\text { personnel à cause } \\
\text { de l'achalandage } \\
\text { - Le cinéma } \\
\text { apporte beaucoup } \\
\text { de clientèle }\end{array}$ & $\begin{array}{l}\text { La variété des clients } \\
\text { Les nouvelles familles } \\
\text { sont plus aisées } \\
\text { économiquement }\end{array}$ & $\begin{array}{l}\text { - Il y a un danger } \\
\text { d'augmentation } \\
\text { des taxes } \\
\text { Augmentation } \\
\text { des coûts des } \\
\text { locaux peu à } \\
\text { peu }\end{array}$ \\
\hline Résidents & $\begin{array}{l}\text { - Il y a une grande } \\
\text { stabilité } \\
\text { résidentielle } \\
\text { C'est excellent, la } \\
\text { présence des } \\
\text { jeunes familles } \\
\text { - Avec les jeunes } \\
\text { familles, on se } \\
\text { sent accompagné } \\
\text { - Les aînés partent } \\
\text { tranquillement } \\
\text { sans être chassés }\end{array}$ & $\begin{array}{l}\text { C'est l'augmenta- } \\
\text { tion normale des } \\
\text { prix à Montréal } \\
\text { Reprise de la } \\
\text { valeur } \\
\text { patrimoniale } \\
\text { - Garantie des } \\
\text { revenus pour le } \\
\text { futur } \\
\text { Est un peu } \\
\text { exagérée }\end{array}$ & $\begin{array}{l}\text { - Il ne reste pas de } \\
\text { locaux } \\
\text { commerciaux } \\
\text { - Les commerces } \\
\text { sont plus beaux }\end{array}$ & $\begin{array}{l}\text { Les nouveaux arrivants } \\
\text { sont des gens qui } \\
\text { consomment de la culture } \\
\text { Les nouveaux résidents } \\
\text { améliorent le quartier } \\
\text { Il n'y a pas de vandalisme } \\
\text { Nous sommes contents } \\
\text { du changement } \\
\text { Les résidents embellissent } \\
\text { plus le quartier } \\
\text { Les coûts des loyers sont } \\
\text { raisonnables } \\
\text { Le parc locatif n'est pas } \\
\text { affecté }\end{array}$ & $\begin{array}{l}\text { L'impossibilité } \\
\text { d'acheter une } \\
\text { propriété dans } \\
\text { le quartier à } \\
\text { cause des prix } \\
\text { élevés } \\
\text { Les enfants ne } \\
\text { pourront pas } \\
\text { continuer à } \\
\text { vivre dans le } \\
\text { quartier, ils ne } \\
\text { pourront pas y } \\
\text { acheter }\end{array}$ \\
\hline
\end{tabular}

Tableau 2- Perception des acteurs sur les transformations du quartier Source : Angulo, 2019, p. 181

\subsection{La protection communautaire contre la gentrification}

Il existe aussi dans le quartier des groupes communautaires, soit des comités de logement, des tables de quartier et des regroupements culturels, qui font un travail de veille en ce qui concerne les éventuelles menaces au cadre de vie du quartier. Ce sont des organismes communautaires qui ont pour mandat la défense des droits des citoyens. Cela semble permettre de protéger l'équilibre dans le système d'acteurs du quartier.

Les résidents du quartier ne semblent pas inquiets pour leur avenir, même s'ils sont conscients des menaces qui pourraient nuire à leurs conditions de vie. Le défi, selon eux, est de conserver leurs acquis sociaux et communautaires. Les résidents ne craignent pas le développement d'un processus de gentrification dans leur quartier. Quand ils y réfèrent, ils le font en regard d'autres quartiers dans l'arrondissement. Un des répondants parle ainsi de la gentrification : «Dans certains secteurs du quartier, oui, dans certains coins d'Angus, à cause du type de maisons, ça convient à des familles branchées, qui font comme des îlots. Mais pas dans le quartier ici; c'est équilibré. » (Entrevue no 1, acteur culturel, 2017)

Les entrevues nous indiquent que les résidents considèrent que le quartier est stable, même s'il traverse un processus de transformation. Il y a de nouvelles familles qui arrivent et apportent un certain rajeunissement au quartier, sans bouleverser la structure démographique des lieux. Les résidents considèrent que la présence des nouvelles familles est positive pour le quartier et la voient comme un retour à un équilibre brisé dans les décennies 1980 et 1990. C’est à ce moment-là qu'il y a eu un départ massif des familles, à cause de la crise économique. 
En ce qui concerne les changements économiques récents, les résidents apprécient le regain de l'activité commerciale des lieux, surtout sur la rue Beaubien. Ils perçoivent ces changements comme positifs pour la vitalité économique du quartier. De plus, l'ensemble des acteurs considère que le Cinéma Beaubien a une grande influence sur l'évolution socioéconomique du quartier.

En ce qui concerne les changements relatifs à la perception des acteurs sur le cadre de vie et le niveau de satisfaction quant au fait d'habiter le quartier, les opinions des résidents sont unanimes : ils sont très satisfaits de leur quartier et de la qualité de vie qu'il leur offre. Les résidents soulignent avec fierté la présence et la contribution du Cinéma Beaubien comme le centre et le moteur de l'activité qui se développe dans le quartier.

Au sujet des perceptions des résidents concernant les éléments les plus intangibles, comme l'identité territoriale, les entrevues montrent que l'ensemble des résidents ressent une grande fierté et un attachement très fort au quartier. L'image villageoise du quartier évoquée par les répondants synthétise bien la perception que les résidents ont du quartier et leur sentiment identitaire à son égard. Ce sentiment d'appartenance s'appuie aussi sur une histoire de mobilisation citoyenne pour préserver les acquis culturels, dont le Cinéma Beaubien, ce qui a renforcé leur identité.

\subsection{L'action culturelle comme facteur de développement local}

La plupart des acteurs du milieu partagent une vision selon laquelle la culture occupe une place centrale en tant que facteur pouvant apporter une contribution au développement de la communauté locale. Pour les acteurs locaux, la culture est omniprésente dans toutes les sphères de la vie des individus et elle est indissociable du développement local. Elle est un ciment de la cohésion sociale et un important levier économique. S'ils n'utilisent pas les mêmes expressions pour la définir, tous s'accordent pour dire qu'elle est fondamentale. Selon les répondants aux entrevues, les initiatives culturelles peuvent jouer un rôle important comme vecteur du dynamisme social :

[La culture] joue un rôle essentiel. Des fois, on ne fait pas toujours le lien avec le développement économique, mais elle joue un rôle primordial. La culture, je la vois dans un sens large. Ça peut générer beaucoup : des rassemblements populaires, un sentiment d'appartenance au quartier. La culture peut démarrer de nouvelles initiatives. Elle peut être aussi un catalyseur. Ça joue un rôle important à tous les niveaux. (Entrevue no 8, acteur public, 2016)

La culture joue un rôle de médiateur social et d'inclusion, notamment à l'endroit des nouveaux arrivants. Les activités avec les enfants, les séances de cinéma à ciel ouvert ainsi que la réduction des prix des billets pour diverses catégories de public, entre autres choses, permettent l'accès à la culture pour les résidents du quartier.

Les répondants s'accordent pour dire que la culture a des effets structurants sur le territoire. À cet égard, plusieurs aspects sont soulignés par les répondants, et la plupart d'entre eux défendent une approche reposant sur une culture de proximité. Dans ce sens, le directeur du Cinéma Beaubien soutient que la culture est un vecteur fort du développement économique et social local :

Il faut qu'il y ait aussi une proximité pour la culture comme pour d'autres activités de la vie de tous les jours. Ça fait partie de la qualité de vie et ça donne de la couleur à chacun des quartiers. (Entrevue no 1, acteur culturel, 2017)

\section{Conclusion}

Le Cinéma Beaubien est devenu l'un des emblèmes du développement du quartier. Son action dans le territoire a constitué un élément essentiel dans la reconstruction du tissu local, qui montrait de forts signes de dévitalisation à la fin des décennies 19801990. La saga de sa relance a insufflé de nouvelles forces aux acteurs et aux résidents, lesquels y voient un facteur d'amélioration du milieu de vie que représente le quartier.

Notre étude montre la capacité des initiatives locales de type culturel à répondre aux changements économiques globaux qui affectent l'économie locale, surtout sur les territoires qui ont vécu longtemps en association avec l'activité industrielle. Ce type d'activité a été lourdement touché par la crise du fordisme, ce qui a provoqué le désarroi des résidents des quartiers qui en dépendaient. La culture, plus concrètement les activités culturelles et créatives, constitue l'un des axes choisis par les organismes locaux pour compenser le déclin des activités manufacturières. 
Nous avons montré que des organismes bien enracinés localement comme le Cinéma Beaubien, en mobilisant des ressources multiples, sont capables de générer des effets positifs importants et durables. Le Cinéma Beaubien a participé à un processus qui a contribué à renverser la tendance au déclin du quartier pour le transformer en un endroit dont les résidents sont fiers. De plus, avec le temps, il a renforcé l'identité territoriale ainsi que le sentiment d'appartenance des résidents. Enfin, l'effet du cinéma déborde largement le champ du cinéma et de la culture. En tant qu'initiative communautaire, la relance du cinéma s'inscrit dans un vaste mouvement où interagissent plusieurs acteurs, lesquels ont mobilisé le capital socioterritorial existant à Rosemont pour viabiliser le projet. De même, le fait d'avoir utilisé l'économie sociale comme stratégie de développement a favorisé la dimension collective de l'entreprise ainsi que son ancrage dans l'économie locale. En même temps, le Cinéma Beaubien a inscrit le quartier dans des réseaux plus larges, à l'échelle de Montréal et du Québec.

En ce qui concerne certains effets négatifs possibles, notre recherche apporte un éclairage en regard du phénomène de gentrification. Plusieurs théoriciens (Smith, 1996; Glass, 1963) argumentent que les activités culturelles et artistiques constituent le premier jalon conduisant à l'augmentation des valeurs foncières, ce qui constitue un déclencheur des processus de gentrification. La rente potentielle stimule les investissements immobiliers, ce qui provoque l'éviction des résidents de longue date et l'arrivée d'une classe aisée de résidents.

Or, les résultats de notre recherche invitent à nuancer cette vision. Ainsi, notre recherche ouvre la voie à de nouvelles interprétations développées par
Ghaffari, Klein et Angulo Baudin (2018), qui postulent que, sous certaines conditions, les activités culturelles et créatives peuvent avoir des effets positifs pour les résidents et, surtout, peuvent éviter les effets négatifs de la gentrification, du moins à court ou moyen terme, si les organismes locaux sont vigilants à cet égard. La relance du Cinéma Beaubien a généré un processus social de revitalisation mené par les acteurs locaux. Ce processus a été amorcé par l'action collective, et non par le capital privé. Cet aspect protège, pour le moment, les résidents des dérives observables dans la plupart des processus de revitalisation associés à la culture.

Notre recherche a démontré aussi que le type de gouvernance exercé par du leadership partagé où concourent plusieurs acteurs a été essentiel à la réussite du projet. La concertation et le partenariat entre les différents acteurs apparaissent comme incontournables pour assurer de bons résultats et la pérennité d'un projet de revitalisation territoriale. L'examen de la trajectoire de l'initiative locale de développement incarnée par le Cinéma Beaubien montre que l'accomplissement de la mission à la fois culturelle et sociale de cette initiative a été largement imputable à l'action de la CDEC, qui a replacé la relance du cinéma dans une trajectoire large de développement communautaire et de partenariat. Or, comme nous l'avons mentionné plus haut, en 2015, la Ville de Montréal, à la suite d'une réforme apportée par le gouvernement du Québec à la gouvernance du développement des territoires, a aboli en 2016 la plupart des CDEC, dont celle de Rosemont-Petite-Patrie. Sans cet encadrement, des initiatives comme celle du Cinéma Beaubien pourront-elles continuer d'être des jalons d'un développement social collectif? Cela reste à voir.

\section{NOTES}

1 Ce texte s'inspire d'une thèse de doctorat en études urbaines soutenue à l'UQAM en 2019 par Wilfredo Angulo, sous la direction de Juan-Luis Klein et de Diane-Gabrielle Tremblay, et intitulée Le développement local par l'intervention culturelle : le cas du Cinéma Beaubien à Rosemont. Les auteurs tiennent à remercier le Conseil de recherche en sciences humaines (CRSH) pour l'appui accordé au programme de recherche La culture dans les quartiers : le tournant culturel des organisations communautaires, dans lequel s'inscrit cette thèse.

2 La CDEC-RPP faisait partie des corporations de développement économique communautaire (CDEC) qui se sont développées à Montréal afin de contrer ou pallier les effets de la crise économique des années 1980. Leur mission était de favoriser le développement économique local dans une perspective citoyenne. Sans écarter les possibilités de s'associer au secteur privé pour développer leurs actions, les CDEC privilégiaient le développement des projets à partir de la perspective de l'économie sociale et du partenariat. Malgré leur succès, reconnu internationalement (Moulaert et MacCallum, 2019), elles ont été dissoutes par la Ville de Montréal en 2015 à la suite d'une réforme de la gouvernance du développement territorial au Québec réalisée sous le signe de l'austérité, par le gouvernement du Québec. 


\section{RÉFÉRENCES}

Ambrosino, C. et Andres, L. (2008). Friches en ville : du temps de veille aux politiques de l'espace. Espaces et sociétés, 134, 37-51. https://doi.org/10.3917/esp.134.0037

Andres, L. (2010). Reconquête culturo-économique des territoires délaissés : de l’importance du temps de veille et de ses acteurs transitoires. Méditerranée, 114, 51-62. https://doi.org/10.4000/mediterranee.4342

Andres, L. et Grésillon, B. (2011). Les figures de la friche dans les villes culturelles et créatives : regards croisés européens. L'Espace géographique, 40(1), 15-30. https:/ / doi.org/10.3917/eg.401.0015

Angulo, W. (2019). Le développement local par l'intervention culturelle : le cas du Cinéma Beaubien à Rosemont (thèse de doctorat). Université du Québec à Montréal. Repéré à https://archipel.uqam.ca/12997/

Boldrini, P. L. et Malizia, M. (2014). Procesos de gentrificación y contragentrificación : Los mercados de Abasto y del Norte en el Gran San Miguel de Tucumán (noroeste argentino) [Gentrification and counter-gentrification processes : The Abasto and Northern Markets in Gran San Miguel de Tucuman (North Western Argentina)]. Revista INVI, 29(81), 157-191. Repéré à http://revistainvi.uchile.cl/index.php/INVI/article/download/811/1147

Collet, A. (2008). Les « gentrifieurs » du Bas Montreuil : vie résidentielle et vie professionnelle. Espaces et sociétés, 132-133(1), 125-141. https://doi.org/10.3917/esp.132.0125

Darchen, S. et Tremblay, D.-G. (2013). The local governance of culture-led regeneration projects: A comparative analysis between Montreal and Toronto. Urban Research \& Practice, 6(2), 140-157. https://doi.org/10.1080/17535069.2013.808433

Díaz-Parra, I. et Rabasco Pozuelo, P. (2013). ¿Revitalización sin gentrificación? Cooperativas de vivienda por ayuda mutua en los centros de Buenos Aires y Montevideo [Revitalisation sans gentrification? Coopératives entraide logement à Buenos Aires et Montevideo centres-villes]. Cuadernos Geográficos, 52(2), 99-118. Repéré à https://revistaseug.ugr.es/index.php/cuadgeo/ article/download/1516/1719

Fontan, J.-M., Klein, J.-L. et Lévesque, B. (dir.). (2003). Reconversion économique et développement territorial. Québec, QC : Presses de l’Université du Québec.

Fontan, J.-M., Klein, J.-L. et Tremblay, D.-G. (2005). Innovation socioterritoriale et reconversion économique : le cas de Montréal. Paris, France : L'Harmattan.

Ghaffari, L. (2020). Pour une gentrification socialement acceptable : le cas d'Hochelaga-Maisonneuve à Montréal et Madeleine-Champ-de-Mars à Nantes (Thèse de doctorat à paraitre). Université du Québec à Montréal, Montréal, QC.

Ghaffari, L., Klein, J.-L. et Angulo Baudin, W. (2018). Toward a socially acceptable gentrification: A review of strategies and practices against displacement. Geography Compass, 12(2), 1-15. https://doi.org/10.1111/gec3.12355

Glass, R. (1963). Introduction to London: Aspects of change. Londres, R.-U. : Center for Urban Studies.

Hall, P. (2000). Creative cities and economic development. Urban Studies, 37(4), 639-649. https://doi.org/10.1080\%2F00420980050003946

Hutton, T.-A. (2008). The new economy of the inner city: Restructuring, regeneration and dislocation in the 21st century metropolis. Londres, R.-U.: Routledge.

Janoschka, M. et Sequera, J. (2014). Procesos de gentrificación y desplazamiento en América Latina: Una perspectiva comparativista. Dans J. J. Michelini (dir.), Desafios metropolitanos: Un diálogo entre Europa y América Latina (p. 82-104). Madrid, Espagne : Catarata.

Klein, J.-L. et Morrissette, P. (2014). Le développement économique communautaire et la cohésion sociale à Montréal : une recherche partenariale sur l'apport des CDEC. Cabiers du Centre de recherche sur les innovations sociales (CRISES), ET1303. Repéré à http://crises.uqam.ca/upload/files/publications/etudes-theoriques/Cahier_CRISES_ET1303.pdf

Klein, J.-L. et Shearmur, R. (dir.). (2017). Montréal : la cité des cités. Québec, QC : Presses de l'Université du Québec.

Klein, J.-L., Tremblay, D.-G., Sauvage, L., Ghaffari, L. et Angulo, W. (2019). Cultural initiatives and local development: A basis for inclusive neighborhood revitalization. Urban Planning, 4(1), 78-90. http://dx.doi.org/10.17645/up.v4i1.1658

Ley, D. (1994). Gentrification and the politics of the new middle class. Environment and Planning D: Society and Space, 12(1), 53-74. https://doi.org/10.1068/d120053

Moulaert, F. et MacCallum, D. (2019). Advanced introduction to social innovation. Cheltenham, R.-U. : Edward Elgar. 
Rius-Ulldemolins, J. et Posso-Jiménez, L. (2016). Cultura, transformación urbana y empoderamiento ciudadano frente a la gentrificación : Comparación entre el caso de Getsemaní (Cartagena de Indias) y el Raval (Barcelona). EURE, 42(126), 97-122. http://dx.doi.org/10.4067/S0250-71612016000200005

Sabatini, F., Darella-Roble, M. et Vásquez, H. (2009). Gentrificacion sin expulsión, o la ciudad latinoamericana en una encrucijada histórica. Revista 180, 24, 18-25. Repéré à http://www.revista180.udp.cl/index.php/revista180/article/view/266/262

Scott, A. J. (2000). The cultural economy of cities. Londres, R.-U.: SAGE.

Scott, A. J. (2014). Villes et régions du capitalisme cognitif et culturel. L’Espace géographique, 43(3), 215-226. https://doi.org/10.3917/eg.433.0215

Scott, A. J. et Leriche, F. (2005). Les ressorts géographiques de l'économie culturelle : du local au mondial. L'Espace géographique, 34(3), 207-222. https://doi.org/10.3917/eg.343.0207

Smith, N. (1996). The new urban frontier: Gentrification and the revanchist city. Oxford, R.-U. : Routledge.

Tremblay, D.-G, Klein, J.-L. et Fontan, J.-M. (2016). Initiatives locales et développement socioterritorial (2e éd.). Québec, QC : TÉLUQ et Presses de l’Université du Québec.

Tremblay, D.-G., Klein, J.-L. et Rochman, J. (2014). Le développement économique communautaire et la cohésion sociale : le cas du cinéma Beaubien à Montréal. Cabiers du CIRTES (hors-série), 4, 21-40.

Repéré à https://pdfs.semanticscholar.org/cf39/a5b8eaa5a40f975be7a52905bec8ae2428a5.pdf 